\title{
ANÁLISE DOS ACIDENTES COM EMBARCAÇÕES DE PESCA E SUA RELAÇÃO COM EVENTOS METEOCEANOGRÁFICOS NO BRASIL

\section{Fabiano Duarte Rosa ${ }^{1}$ e Thiago Pereira Alves ${ }^{1}$}

\begin{abstract}
Resumo
A pesca comercial está entre as atividades laborais mais perigosas do mundo. A escassez nas capturas obrigou a frota pesqueira a se deslocar para áreas mais distantes do litoral e por períodos de tempo mais longos, tornando as viagens de pesca mais suscetíveis às mudanças bruscas nas condições meteorológicas e consequentemente, no estado do mar. Estudos têm demonstrado a correlação entre a ocorrência de eventos meteoceanográficos adversos com o aumento do risco de ocorrência de acidentes. Esse tipo de abordagem no Brasil é ainda incipiente, o que motivou o desenvolvimento da presente proposta, que objetiva criar subsídios para a Autoridade Marítima estabelecer ferramentas de prevenção de acidentes com barcos de pesca. O método proposto prevê a o tratamento e análise dos registros históricos dos Inquéritos Administrativos e Fatos da Navegação(IAFN) da Autoridade Marítima para o período de 2015a2020. Os avisos de Mau Tempo emitidos pela Marinha do Brasil para o mesmo período serão utilizados como parâmetro subjetivo das condições meteoceanográficas, e sua distribuição espacial e temporal para o litoral do Brasil deverá ser confrontada com as informações dos acidentes registrados. Os resultados preliminares indicam uma tendência de redução no número de acidentes para o período em análise. Os acidentes de maior ocorrência são os naufrágios e situação de homem ao mar. Este últimoapresentou a maior letalidade associada. A maior incidência no número de acidentes está relacionada com a região do $5^{\circ}$ Distrito Naval (PR, SC e RS), o que pode estar relacionado a maior ocorrência de fenômenos adversos, como a passagem de frentes frias e ciclones extratropicais.
\end{abstract}

\section{Palavras-chave}

pesca comercial, acidentes com embarcações de pesca, eventos meteoceanográficos adversos 


\section{INTRODUÇÃO}

A atividade de pesca comercial está entre as profissões mais perigosas do mundo de acordo com a Organização Internacional do Trabalho (International Labour Organization - ILO). Aproximadamente 24.00 pescadores profissionais morrem todos os anos em virtude do trabalho nesta atividade (ILO, 1999). De acordo com Wu, Pelot, and Hilliard (2005) as condições que os pescadores enfrentam diariamente, convivendo com a possibilidade de perda de vida, estão muito além dos riscos associados aos trabalhos realizados em terra.

Historicamente, as capturas obtidas próximas ao litoral têm apresentado quedas significativas de produção, em razão do estado de sobre-exploração dos principais recursos pesqueiros (DIAS NETO, 2001). Essa tendência de queda na produtividade incentivou o redirecionamento da frota pesqueira para áreas mais distantes da costa, e por períodos de tempo mais longos, aumentando a exposição dos pescadores aos riscos inerentes à atividade. O distanciamento da linha de costa tornou-se um problema, uma vez que a frota pesqueira é predominantemente de pequena escala, composta por embarcações que não foram construídas para as condições atuais de operação (mar aberto), e não oferecem características dignas de segurança e habitabilidade aos seus tripulantes para permanecer por muito tempo no mar (FAO, 2020).

Estudos da Organização Internacional do Trabalho demonstram para a atividade de pesca um índice anual de fatalidade de 80 mortes para cada 100.000 pescadores em todo o mundo (ILO, 1999). As consequências da perda de vida na atividade pesqueira têm um impacto profundo sobre a família do trabalhador da pesca. Nos países em desenvolvimento, estas consequências podem ser devastadoras, uma vez que o Estado não oferece políticas de bem-estar social para alentar a família do pescador, condenando viúvas e filhos a uma condição de miséria pela falta de uma fonte alternativa de renda (FAO, 2020).

Nesse sentido, o presente trabalho estabelece uma investigação dos registros de acidentes com embarcações de pesca no litoral do Brasil e sua relação com os eventos meteoceanográficos adversos à navegação. Para tanto, pretende-se avaliar distribuição da frequência dos diversos tipos de acidentes com embarcações de pesca, identificando o local da ocorrência, o período de ocorrência, as características físicas das embarcações e a letalidade dos eventos.

O objetivo geral do trabalho consiste na comparação entre a ocorrência dos acidentes com barcos de pesca e a frequência da publicação dos Avisos de Mal Tempo do Centro de Hidrografia da Marinha do Brasil, no período de 2015 a 2020, permitindo compreender, de forma subjetiva, a influência dos eventos meteoceanográficos como fator de risco à segurança das operações de pesca comercial no litoral do Brasil.

Como objetivos específicos, o trabalho propõe:

\footnotetext{
${ }^{1}$ Programa de Mestrado Profissional em Clima e Ambiente, Instituto Federal de Santa Catarina (IFSC)

Email: thiago.alves@ifsc.edu.br (Thiago Pereira Alves)
}

Corresponding author:

Fabiano Duarte Rosa, Programa de Mestrado Profissional em Clima e Ambiente, Instituto Federal de Santa Catarina (IFSC)

Email: fabiano.duarte@ifsc.edu.br 
a) Investigar as ocorrências de acidentes com embarcações de pesca comercial a partir do registro de Inquéritos Administrativos sobre Acidentes e Fatos da Navegação da Marinha do Brasil;

b) Avaliar a distribuição espacial e temporal dos acidentes com embarcações de pesca no litoral do Brasil, considerado a tipologia e a gravidade das ocorrências;

c) Comparar a ocorrência dos acidentes envolvendo embarcações de pesca com o registro de eventos meteoceanográficos adversos à navegação;

d) Determinar os períodos do ano e as áreas do litoral do Brasil menos favoráveis à segurança da navegação.

\section{REVISÃO BIBLIOGRÁFICA}

De acordo com a Organização Internacional para Agricultura e Alimentação (Food and Agriculture Organization - FAO) os padrões de segurança a bordo de embarcações de pesca recebem menos atenção durante o trabalho realizado em circunstâncias perigosas e estressantes, como por exemplo, jornadas laborais noturnas ou sob condições meteorológicas adversas (FAO, 2020).

Wang et al. (2021) avaliaram os fatores que influenciam a gravidade dos acidentes marítimos em todo o mundo, e indicam a necessidade de uma atenção especial para o potencial de risco para embarcações de pesca, operando em condições meteorológicas adversas e distante dos portos.

Nos Estados Unidos e no Canadá, estudos de D. Jin (2014), e Rezaee, Pelot, Ghasemi (2016), respectivamente, demonstraram que grande parte dos acidentes ocorridos com embarcações de pesca comercial estão associados às condições meteoceanográficas adversas.

Sistemas meteorológicos influenciam na precipitação e na ocorrência de nevoeiros, prejudicando a visibilidade na navegação e acarretando em risco de colisão ou abalroamento. Ventos fortes influenciam na altura significativa das ondas, tornando as condições do mar muito perigosas para a navegação (Guedes, Soares, Bitner-Gregersen, \& Antão, 2001) e consequentemente, em risco de naufrágio (JIN, KITE-POWELL, TALLEY, 2001).

$\mathrm{Na}$ costa brasileira, incidentes de naufrágio foram influenciados pela velocidade do vento e altura de ondas, principalmente nas regiões Sudeste e Sul, com maior ocorrência entre os meses de março e agosto (FUENTES, BITENCOURT E FUENTES, 2013), possivelmente estariam relacionados com sistemas meteorológicos, como frentes frias e ciclones extratropicais.

Além dos fatores de risco relacionados ao meio ambiente, Rezaee, Pelot e Ghasemi (2016) sugerem que os acidentes na atividade de pesca estão associados com seus aspectos intrínsecos, como as características físicas das embarcações, a experiência dos pescadores e o ordenamento pesqueiro.

Apesar da grande incidência de acidentes fatais associados com a atividade de pesca em todo o mundo, as informações existentes são insuficientes e não apresentam equivalência entre os paísespara efeitos de estudos e comparações (PEREZ-LABAJOS et al., 2006).

No Brasil, os trabalhos acadêmicos que tratam do tema ainda são escassos. De acordo com Luz (2017), embora existam legislações voltadas à segurança da navegação, a carência de estudos estatísticos envolvendo as características das embarcações e os acidentes ocorridos motivam pesquisas nesta área. A análise dos registros históricos dos acidentes com embarcações de pesca representa uma importante ferramenta de gestão para avaliar a performance de segurança na atividade (JIN e THUNBERG, 2005). 


\section{METODOLOGIA}

A fim de alcançar os objetivos propostos no presente estudo, foram determinadas duas etapas de avaliação para posterior investigação das correlações. A primeira etapa de avaliação corresponde a caracterização dos acidentes envolvendo embarcações de pesca no Brasil. A segunda etapa consiste na caracterização das condições meteoceanográficas que pudessem interferir na segurança da navegação.

A caracterização dos acidentes com embarcações de pesca foi realizada a partir dos relatórios anuais de Inquéritos Administrativos sobre Acidentes e Fatos da Navegação - IAFN, disponíveis para o período compreendido entre os anos de 2015 e 2020, divulgados pela Marinha do Brasil em seu portal eletrônico $<$ https://www.marinha.mil.br/dpc/acidentes-de-navegacao2> (DPC, 2020).

Os relatórios foram compilados, filtrados, e categorizados, permitindo analisar os acidentes envolvendo embarcações de pesca registradas no Brasil, destacando-se variáveis qualitativas e quantitativas passíveis de serem manipuladas estatisticamente, conforme apresentado a seguir:

a) Nome da embarcação: para efeito de identificação do acidente, sem efeito prático nasanálises;

b) Distrito Naval (DN): o território brasileiro é subdividido em áreas de jurisdição dos Distritos Navais da Marinha do Brasil. A depender da localização de ocorrência do acidente ou fato da navegação, o seu registro estará associado a um determinado Distrito Naval, responsável pelo Serviço de Busca e Salvamento daquela região.

c) Comprimento: refere-se a medida linear horizontal entre os pontos extremos de proa e de popa, expressa em metros.

d) Data de ocorrência do acidente;

e) Descrição da natureza: corresponde a classificação do tipo de acidente ou fato da navegação registrado no IAFN. Para a presente análise, foram elencados exclusivamente os acidentes cuja a ocorrência possa ter influência dos fatores ambientais.

f) Vítimas: expressa a ocorrência de óbitos e desaparecidos (morte presumida) relacionados aos acidentes.

As informações disponíveis foram tabuladas e padronizadas a partir do local de ocorrência(Distrito Naval) e por data (mês/ano), permitindo compreender a distribuição espacial e temporal dos acidentes no período definido, assim como determinar a contribuição de cada tipificação no total observado e a letalidade associada. O software Microsoft Excel foi utilizado para organização e tratamento das informações e Minitab Statistical Software para as análises estatísticas e geração dos gráficos.

A caracterização das condições meteoceanográficas ainda estão em andamento, a partir da organização das informações presentes nos 9.550 Avisos de Mau Tempo emitidos pelo Centro de Hidrografia da Marinha do Brasil (CHM), para a área de Meteorologia Marítima sob responsabilidade do Brasil, identificada como METAREA V. Esses informes correspondem a alertas das condições dotempo e do mar, adversas à segurança da navegação e são emitidos quando houver previsão de uma ou mais das seguintes situações (DHN, 2018):

a) Vento com intensidade superior a 28 nós para zona costeira e 34 nós para as áreas oceânicas.

b) Ondas com altura significativa de 3 metros ou superior na costa e de 4 metros ou superior para as áreas oceânicas;

c) Visibilidade horizontal inferior a $1 \mathrm{~km}$ (nevoeiros);

d) Ondas com altura significativa de 2,5 metros ou superior atingindo a linha de costa;e

e) Formação de ciclones tropicais ou subtropicais. 
Apesar de não se tratar de observações registradas, as previsões contidas nos Avisos de Mau Tempo serão adotadas como parâmetro subjetivo para determinar a frequência de ocorrência dos eventos meteorológicos adversos à segurança a navegação, de forma a caracterizar a sua distribuição mensal por área de ocorrência, no período de 2015 a 2020.

Após conclusão das etapas propostas pelo presente trabalho, será realizada uma análise focada nas possíveis correlações entre os acidentes registrados para as embarcações de pesca e a frequência dos Avisos de Mau Tempo emitidos pela Marinha do Brasil.

\section{RESULTADOS PRELIMINARES E DISCUSSÃO}

Foram registrados pela Autoridade Marítima 718 Inquéritos Administrativos sobre Acidentes e Fatos da Navegação para o período estudado. Deste total, 508 registros $(79,6 \%)$ foram classificados como acidentes marítimos, de acordo com os critérios definidos no Código de Padrões Internacionais e Práticas Recomendadas para uma Investigação de Segurança dos Acidentes e Incidentes Marítimos, aprovado pela Resolução MSC. 255 (84) da Organização Marítima Internacional - IMO (IMO, 2008).

A definição da tipificação dos acidentes procurou conciliar os padrões definidos pela IMO e pela Autoridade Marítima brasileira, uma vez que as planilhas dos IAFN não apresentavam a natureza dos acidentes de forma padronizada. Assim, o presente estudo adotou a seguinte tipificação dos acidentes:

(a) abalroamento ou colisão, (b) acidente com tripulante, (c) água aberta (alagamento), (d) encalhe, (e) homem ao mar, (f) morte de pessoa e (g) naufrágio.

$\mathrm{O}$ total de acidentes registrados resultou na morte e desaparecimento de 308 pessoas no período, o que corresponde a uma razão de 0,54 , ou seja, para cada dois acidentes ocorridos, uma pessoa foi vitimada. O acidente que apresenta a maior incidência são os naufrágios $(35,14 \%)$, seguido da situação de homem ao mar (20,80\%). Contudo, o percentual de vítimas é o mesmo nestes tipos de acidente, o que implica em afirmar que a letalidade das situações de homem ao mar é a maior observada (Tabela 1).

Tabela 1. Registro dos acidentes com embarcações de pesca e a letalidade associada.

\section{Table 1.}

\begin{tabular}{|c|c|c|c|c|c|}
\hline Tipificação & $\begin{array}{l}\mathbf{N}^{\circ} \text { de } \\
\text { eventos }\end{array}$ & $\begin{array}{l}\text { \% de } \\
\text { Contribuição }\end{array}$ & $\begin{array}{l}\text { Víti- } \\
\text { mas }\end{array}$ & $\begin{array}{l}\text { \% do } \mathrm{N}^{\circ} \text { de } \\
\text { vítimas }\end{array}$ & $\begin{array}{l}\text { Vítimas } / n^{\circ} \text { de } \\
\text { eventos }\end{array}$ \\
\hline $\begin{array}{l}\text { Abalroamento ou } \\
\text { colisão }\end{array}$ & 91 & 15,91 & 13 & 4,22 & 0,14 \\
\hline $\begin{array}{l}\text { Acidente com } \\
\text { tripulante }\end{array}$ & 92 & 16,08 & 36 & 11,69 & 0,39 \\
\hline Água aberta & 16 & 2,80 & 1 & 0,32 & 0,06 \\
\hline Encalhe & 21 & 3,67 & 1 & 0,32 & 0,05 \\
\hline Homem ao mar & 119 & 20,80 & 112 & 36,36 & 0,94 \\
\hline Morte de pessoa & 32 & 5,59 & 32 & 10,39 & 1,00 \\
\hline Naufrágio & 201 & 35,14 & 113 & 36,69 & 0,56 \\
\hline Total & 572 & 100,00 & 308 & 100,00 & $\mathbf{0 , 5 4}$ \\
\hline
\end{tabular}

Fonte: elaborado a partir dos relatórios de IAFN da Marinha do Brasil. 
O item morte de pessoa apresenta uma razão vítimas $/ \mathrm{n}^{\circ}$ de acidentes igual a 1 , uma vez que a tipificação corresponde ao óbito em si. Os acidentes relacionados a água aberta (alagamento do casco) e encalhe apresentaram os menores percentuais de contribuição no total de eventos $-2,80 \%$ e $3,67 \%$ respectivamente $-\mathrm{e}$ as menores fatalidades.

O número de ocorrências apresentou uma tendência de redução ao longo do período, com o menor patamar observado para o ano de 2020. O número de vítimas fatais acompanhou esta tendência de redução. Contudo, o menor patamar de óbitos relacionados aos acidentes corresponde ao ano de 2018 (Figura 1.a).

Ao analisar a distribuição espacial dos acidentes (Figura 1.b), é possível observar que a região do $5^{\circ}$ Distrito Naval - DN (PR, SC e RS) apresenta a maior contribuição de eventos, seguido pela região do $3^{\circ}$ $\mathrm{DN}$ (AL, PE, PB, RN, CE) e do $1^{\circ} \mathrm{DN}$ (RJ e ES). As regiões do $6^{\circ} \mathrm{DN}$ (MT e MS) e do $7^{\circ} \mathrm{DN}$ (TO e $\mathrm{GO}$ ) apresentaram as menores contribuições no número total de acidentes, o que pode ser explicado por se tratar de uma atividade de pesca comercial pouco expressiva e realizada em ambientes continentais.

Figura 1 - (a) Registro de vítimas fatais de acidentes com embarcações de pesca ao longo do período analisado e (b) distribuição espacial dos acidentes com embarcações de pesca no Distritos Navais.

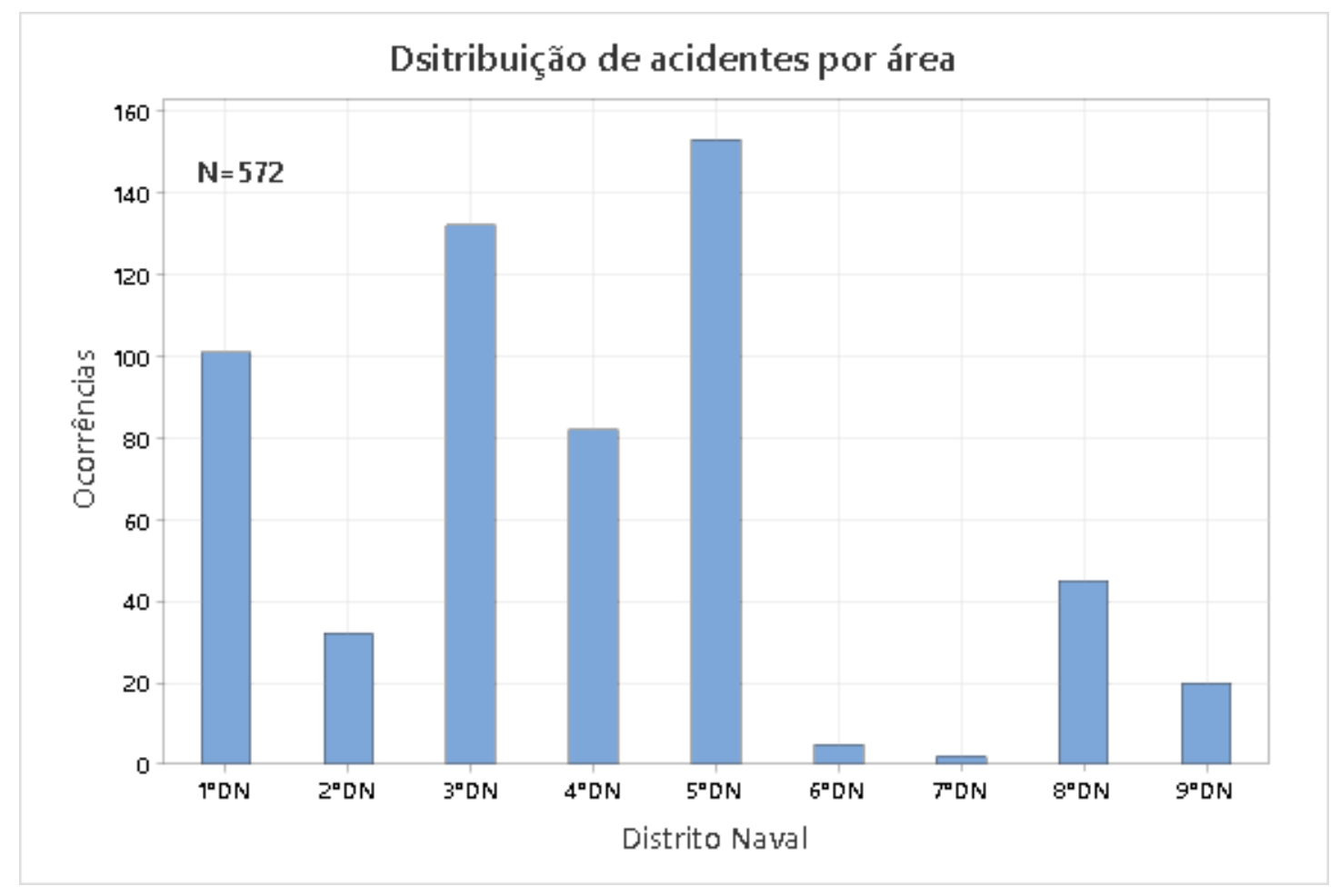

Figure 1.

Fonte: elaborado a partir dos relatórios de IAFN. 


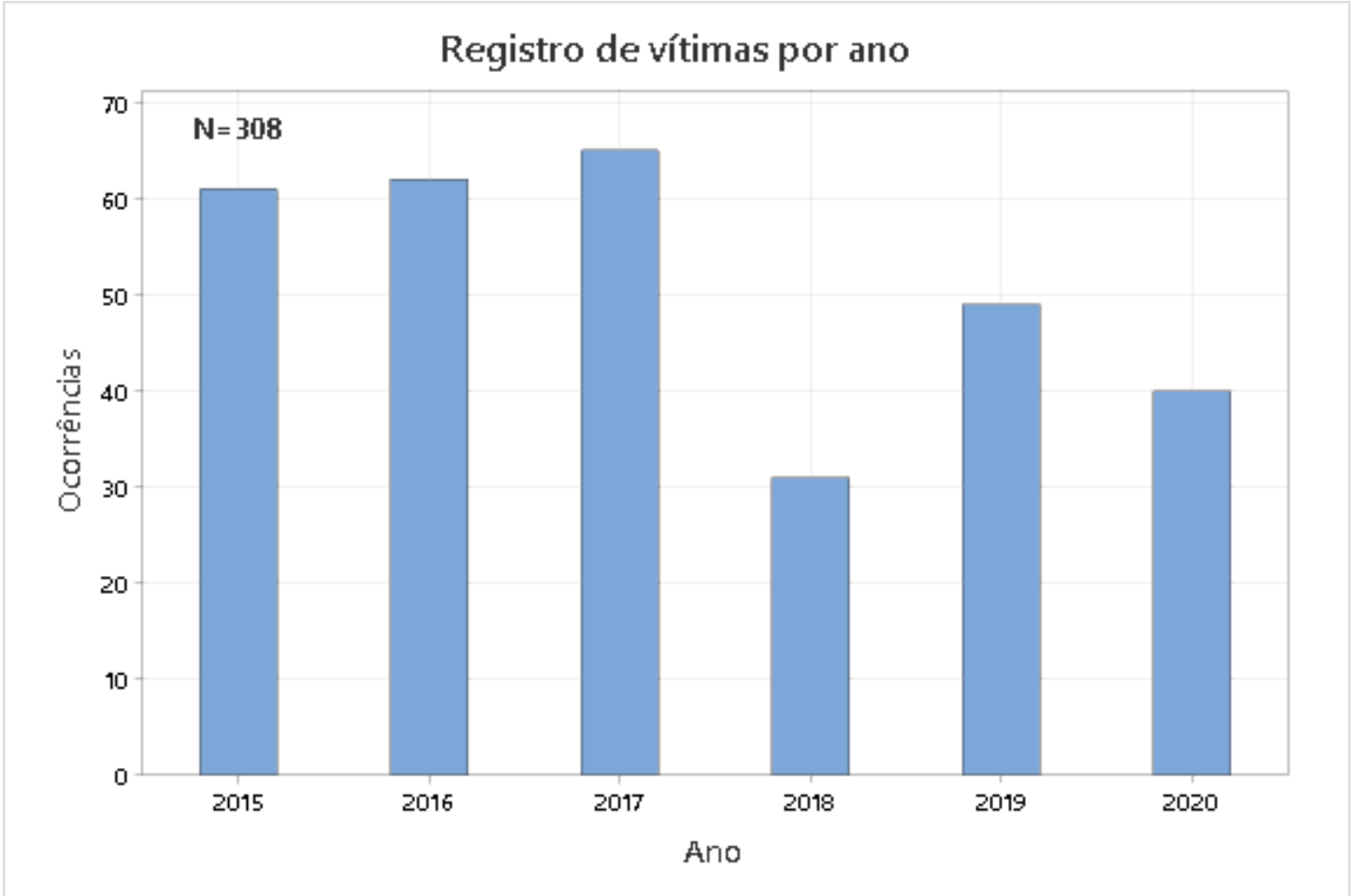

Figure 2.

A maior ocorrência de acidentes registrados para o $1^{\circ} \mathrm{DN}$ (RJ e ES) e $5^{\circ} \mathrm{DN}$ (SC e RS) estão em consonância com o trabalho realizado por Fuentes, Bitencourt e Fuentes (2013) que destaca o papel das condições meteorológicas e oceanográficas sobre os incidentes de naufrágio na costa brasileira, em especial em latitudes mais elevadas. Os valores observados para o $3^{\circ} \mathrm{DN}$ (AL, PE, PB, RN, CE) e $4^{\circ}$ $\mathrm{DN}$ (PI, MA e PA) podem estar associados às características físicas das embarcações empregadas naquela região, uma vez que condições meteorológicas e oceanográficas adversas não são frequentes nas latitudes mais baixas.

\section{CONCLUSÕES}

A maior parte dos acidentes com embarcações de pesca ocorreu nas regiões Sudeste e Sul do Brasil. A queda de tripulante ao mar corresponde ao acidente com a maior letalidade associada. Os registros indicam uma tendência geral de redução do número de acidentes, e respectivamente a redução da ocorrência de vítimas fatais para o período analisado. As informações registradas e divulgadas pela Marinha do Brasil carecem de padronização para efeitos de estudo e comparação. 


\section{References}

DIAS NETO, J. Gestão do uso dos recursos pesqueiros marinhos no Brasil: 2. Ed. Brasília: IBAMA, 2010.

DIRETORIA DE HIDROGRAFIA E NAVEGAÇÃO - DHN. Normas da autoridade marítima para as atividades de meteorologia marítima /NORMAM-19. $1^{\text {a }}$ Revisão, 2018. Disponível em: https://www.marinha.mil.br/dhn/sites/www.marinha.mil.br.dhn/files/normam/NORMAN-19-REV-1-Ed2018-CHM.pdf. Acesso em: 1 fev. 2021.

DIRETORIA DE PORTOS E COSTAS - DPC. Acidentes de Navegação. Disponível em: $<$ https://www.marinha.mil.br/dpc/acidentes-de-navegacao2>. Acesso em: 05 mar. 2020.

FOOD AND AGRICULTURE ORGANIZATION - FAO. The state of fisheries and aquaculture. Roma: FAO, 2008.

FOOD AND AGRICULTURE ORGANIZATION - FAO. Fishing operations: best practices to improve safety at sea in the fisheries sector. Roma: FAO, 2015. Disponível em:

http://www.fao.org/3/i4740e/i4740e.pdf. Acesso em: 1 dez. 2020.

FUENTES, Eduardo Vetromilla; BITENCOURT, Daniel Pires; FUENTES, Márcia Vetromilla. Análise da velocidade do vento e altura de onda em incidente de naufrágio na costa brasileira entre os estados do Sergipe e do Rio Grande do Sul. Revista Brasileira de Meteorologia, v. 28, n. 3, p. 257-266, fev. 2013. Disponível em: http://www.scielo.br/pdf/rbmet/v28n3/03.pdf. Acesso em 15 out. 2019.

GUEDES SOARES, C., BITNER-GREGERSEN, E.M.; ANTÃO, P. Analysis of the frequency of ship accidents under severe North Atlantic weather conditions. Design and operation for Abnormal Conditions II: The Royal Institution of Naval Architects, p. 221-230. Londres, 2001. Disponível em: https://www.researchgate.net/publication/281269983. Acesso em: janeiro de 2020.

INTERNATIONAL LABOUR ORGANIZATION - ILO. Note on the proceedings: Tripartite Meeting on Safety and Health in the Fishing Industry. Organização Internacional do Trabalho. Genebra: ILO, 1999 .

INTERNATIONAL MARITIME ORGANIZATION - IMO. Código de Padrões Internacionais e Práticas Recomendadas para uma Investigação de Segurança dos Acidentes e Incidentes Marítimos (2008). Resolução MSC. 255 (84). Disponível em: https:/www.marinha.mil.br/dpc/sites/ www.marinha.mil.br. dpc/ files/ ResMSC255\%2884\%29COD_INVESTIGACAOACIDENTES.pdf. Acesso em: 01 de fevereiro de 2021. 
JIN, Di; THUNBERGB, Eric. An analysis of fishing vessel accident in fishing areas off the northeastern United States. Safety Science, 43, p. 523-540, fev. 2005. https://doi.org/10.1016/j.ssci.2005.02.005

JIN, Di. The determinants of fishing vessel accident severity. Accident Analysis and Prevention, 66, p. 1-7, jan. 2014. https://doi.org/10.1016/j.aap.2014.01.001

LUZ, H. H. Análise quantitativa dos acidentes de navegação em águas brasileiras entre 2006 e 2015. Trabalho de Conclusão de Curso (Engenharia Naval) - Centro Tecnológico de Joinville, Universidade Federal de Santa Catarina. Joinville. 2017.

PEREZ-LABAJOS, Carlos et al. Analysis of accident inequality of the Spanish fishing fleet. Accident Analysis \& Prevention, v. 38, p. 1168-1175, mai. 2006. https://doi.org/10.1016/j.aap.2006.05.007

REZAEE, Sara; PELOT, Ronald; GHASEMI, Alireza. The effect of extreme weather conditions on commercial fishing activities and vessel incident in Atlantic Canada. Ocean \& Coastal Management, v. 130, p. 115-127, mai. 2016. https://doi.org/10.1016/j.ocecoaman.2016.05.011

WANG, Huanxin et al. An analysis of factors affecting the severity of marine accidents. Reliability Engineering and System Safety, 210, 107513, 2020. https://doi.org/10.1016/j.ress.2021.107513

WU, Yue; PELOT, Ronald P.; HILLIARD, Casey. The effect of weather factors on the severity of fishing boat accidents in Atlantic Canada. Risk management: An International Journal, v. 7, n. 3, p. 21 40, 2005. https://doi.org/10.1057/palgrave.rm.8240217

\section{Author Biographies}

Fabiano Duarte Rosa Discente do Programa de Mestrado Profissional em Clima e Ambiente do Instituto Federal de Santa Catarina (IFSC).

Thiago Pereira Alves Docente do Programa de Mestrado Profissional em Clima e Ambiente do Instituto Federal de Santa Catarina (IFSC). 